\title{
Remote Collaboration in AR and VR using Virtual Replicas
}

\author{
Carmine Elvezio, Mengu Sukan, Ohan Oda, \\ Steven Feiner \\ Dept. Of Computer Science, Columbia University \\ New York, NY 10027 \\ \{carmine,mengu,ohan,feiner\}@cs.columbia.edu
}

\author{
Barbara Tversky \\ Dept. of Human Dev., Teachers College \\ New York, NY 10027 \\ btversky@stanford.edu
}
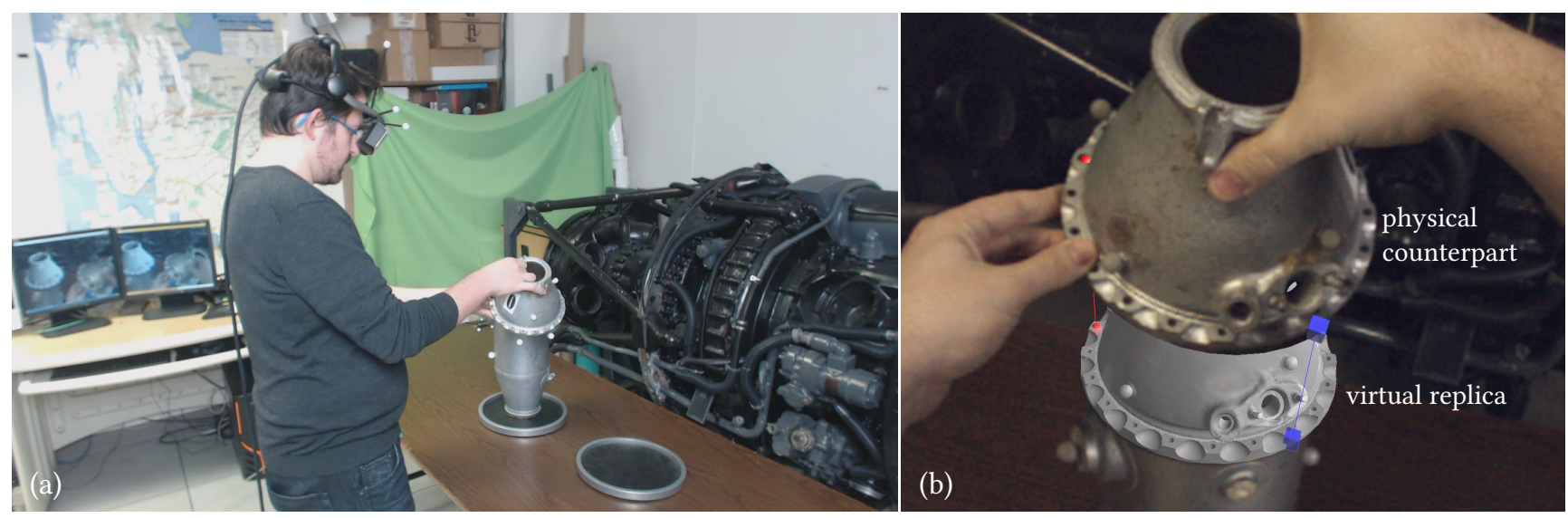

Figure 1: (a) Guided by remote expert, local user wearing tracked see-through HWD places top of aircraft-engine combustion chamber on bottom of chamber. (b) View seen by local user in AR (imaged through video see-through display): Remote expert has placed virtual replica of chamber top on physical chamber bottom. Pairs of metaobject annotations on virtual replica and physical chamber top are linked with color-coded red and blue lines to show correspondence and assist in correct alignment.

\begin{abstract}
In many complex tasks, a remote subject-matter expert may need to assist a local user, to guide their actions on objects in the local user's environment. However, effective spatial referencing and action demonstration in a remote physical environment can be challenging. We demonstrate an approach that uses Virtual Reality (VR) or Augmented Reality (AR) for the remote expert, and AR for the local user, each wearing a stereo head-worn display (HWD). Our approach allows the remote expert to create and manipulate virtual replicas of physical objects in the local environment to refer to parts of those physical objects and to indicate actions on them. This can be especially useful for parts that are occluded or difficult to access. The remote expert can demonstrate actions in 3D by manipulating virtual replicas, supported by constraints and annotations, and point in 3D to portions of virtual replicas to annotate them.
\end{abstract}

\section{CCS CONCEPTS}

-Computing methodologies $\rightarrow$ Mixed / augmented reality; Virtual reality; $\bullet$ Human-centered computing $\rightarrow$ Collaborative interaction;

Permission to make digital or hard copies of part or all of this work for personal or classroom use is granted without fee provided that copies are not made or distributed for profit or commercial advantage and that copies bear this notice and the full citation on the first page. Copyrights for third-party components of this work must be honored For all other uses, contact the owner/author(s).

SIGGRAPH '17 VR Village, Los Angeles, CA, USA

(C) 2017 Copyright held by the owner/author(s). 978-1-4503-5013-6/17/07 ..\$15.00 DOI: $10.1145 / 3089269.3089281$

\section{KEYWORDS}

Collaborative mixed/augmented reality; 3D referencing techniques; remote task assistance; remote guidance; assembly; maintenance.

ACM Reference format:

Carmine Elvezio, Mengu Sukan, Ohan Oda, Steven Feiner and Barbara Tversky. 2017. Remote Collaboration in AR and VR using Virtual Replicas. In Proceedings of SIGGRAPH '17 VR Village, Los Angeles, CA, USA, fuly 30 August 03, 2017, 2 pages.

DOI: $10.1145 / 3089269.3089281$

\section{INTRODUCTION}

Task guidance has been an active topic in the field of augmented reality (AR), with applications to a wide range of domains, including the operation, assembly, maintenance, and repair of equipment (e.g., [Bottecchia et al. 2010; Gauglitz et al. 2014; Henderson and Feiner 2011; Sukan et al. 2016; Tecchia et al. 2012]). One especially productive approach to task guidance involves a remote subject-matter expert assisting a local user. Traditional approaches to remote guidance using voice or video limit how the remote expert can instruct the local user, especially for operations that require 3D spatial referencing and action demonstration. Language describing spatial locations and actions in space can be ambiguous or vague, leading to confusion and error (e.g., [Heiser et al. 2004]). In contrast, AR enables a remote expert to directly interact with the local environment for 3D spatial referencing and action demonstration and allows a local user to view live instructions directly overlaid on their environment (Figure 1). 


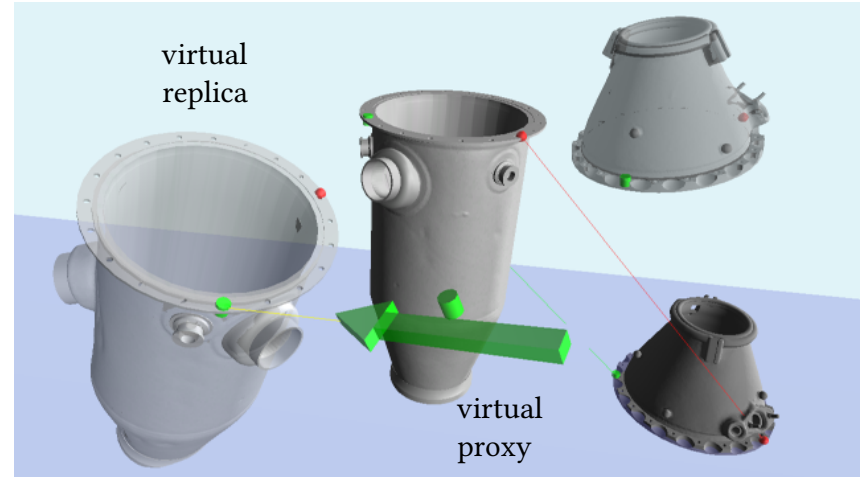

Figure 2: Annotation being performed by remote expert in VR: Expert places 3D annotations (green cylinders) on semitransparent virtual replica, specifying contact points. The same annotations appear on virtual proxy corresponding to virtual replica and physical counterpart in local user's AR view.

\section{INTERACTION AND VISUALIZATION TECHNIQUES}

We have developed and evaluated 3D interaction and visualization techniques [Oda et al. 2015] that are based on the ways an expert guides a novice when co-located, by pointing to relevant places or demonstrating appropriate actions. Our techniques, which we demonstrate here, are intended for use by a remote expert and local user, who each wear a head-tracked stereo head-worn display (HWD). Inspired in part by Voodoo-Doll interaction [Pierce et al. 1999], we allow the remote expert to create virtual replicas of physical counterparts present in the local user's environment (Figure 1b). The virtual replicas can be used to demonstrate how to position and orient the physical counterparts (Figure 1b). The remote expert can also point to locations on the virtual replicas and present these to a local user in AR (Figure 2).

Our techniques are designed to alleviate time-consuming and error-prone aspects of the interaction for both the remote expert and the local user. For example, the remote expert can define simple constraints to allow fast and precise placement of virtual replicas. Similarly, to help the local user mentally map the position and orientation of a virtual replica to its physical counterpart, we render a set of metaobjects on a virtual replica and its physical counterpart (Figure $1 \mathrm{~b}$ ). The remote expert is presented with a virtual representation (Figure 2) that uses virtual models of the 6DOFtracked physical objects. The remote expert can freely navigate among and interact with these virtual models, whose poses can be efficiently transmitted.

\section{DEMONSTRATION}

While a paper describing and formally evaluating an earlier version of this system and its techniques was presented at ACM UIST 2015 [Oda et al. 2015], this will be the first live public demonstration of the system, and will allow attendees to try it firsthand. In our demonstration, a local user interacts with physical objects that are replicated virtually for the remote expert. In the example shown in Figure 1 (created with an earlier version of our system using a Canon HM-A1 stereo video-see-through HWD), the local user assembles two parts of an aircraft engine combustion chamber by following instructions provided by the remote expert. We will use this task domain and/or an additional domain created for the SIGGRAPH demonstration.

In our demonstration, an attendee acting as the local user will wear a head-tracked Meta 2 stereo optical-see-through HWD. Another attendee acting as the remote expert will also wear a headtracked stereo HWD. We will allow attendees playing the remote expert to choose between wearing an optical-see-through HWD (a second Meta 2) and using their hands for manipulation, or wearing an opaque VR HWD (Oculus Rift) and using its tracked controller (Oculus Touch). 6DOF tracking will be performed by a multi-camera NaturalPoint OptiTrack system and Oculus tracking.

\section{CONCLUSIONS}

We present a demonstration of 3D interaction and visualization techniques in AR and VR that efficiently support interaction in remote assistance scenarios, where both users wear tracked HWDs. Our techniques allow a remote expert to create and use virtual replicas of physical objects to guide a local user performing a task with those objects. A formal user study showed the advantages of these techniques over alternatives [Oda et al. 2015].

\section{ACKNOWLEDGMENTS}

This material is based on work supported in part by the National Science Foundation under Grants IIS-0905569, IIS-0905417, IIS-1514429, and IIS-1513841. We appreciate the generosity of Meta for lending the Meta 2 Development Kits and Canon U.S.A., Inc. for lending the HM-A1 head-worn display. Feiner is Lead Advisor to Meta. We thank Steve Henderson for refurbishing the aircraft engine combustion chamber.

\section{REFERENCES}

S. Bottecchia, J. Cieutat, and J. Jessel. 2010. T.A.C: Augmented Reality System for Collaborative Tele-assistance in the Field of Maintenance Through Internet. In Proc. ACM AH. 14:1-7.

S. Gauglitz, B. Nuernberger, M. Turk, and T. Höllerer. 2014. World-Stabilized Annotations And Virtual Scene Navigation For Remote Collaboration. In Proc. ACM UIST. 449-459.

J. Heiser, B. Tversky, and M. Silverman. 2004. Sketches for and from Collaboration. Visual and spatial reasoning in design III 3 (2004), 69-78.

S. Henderson and S. Feiner. 2011. Augmented Reality in the Psychomotor Phase of a Procedural Task. In Proc. IEEE ISMAR. 191-200.

O. Oda, C. Elvezio, M. Sukan, S. Feiner, and B. Tversky. 2015. Virtual Replicas for Remote Assistance in Virtual and Augmented Reality. In Proc. ACM UIST. 405-415. DOI : https://doi.org/10.1145/2807442.2807497

J.S. Pierce, B.C. Stearns, and R. Pausch. 1999. Voodoo Dolls: Seamless Interaction at Multiple Scales in Virtual Environments. In Proc. ACM i3D. 141-145.

M. Sukan, C. Elvezio, S. Feiner, and B. Tversky. 2016. Providing Assistance for Orienting 3D Objects Using Monocular Eyewear. In Proceedings of the 2016 Symposium on Spatial User Interaction (SUI '16). ACM, 89-98.

F. Tecchia, L. Alem, and W. Huang. 2012. 3D Helping Hands: A Gesture Based MR System for Remote Collaboration. In Proc. ACM VRCAI. 323-328. 